\title{
Diseño hidrológico de zanjas de infiltración en el secano costero e interior de las regiones semiáridas de Chile
}

\author{
Hydrologic design of infiltration ditches in coastal and dry lands of Chilean semiarid regions \\ Roberto Pizarro Tapia**, Juan Pablo Flores Villaneloa, Claudia Sangüesa Poola, \\ Enzo Martínez Araya ${ }^{a}$, Lastenia León Gutiérrez ${ }^{\mathrm{a}}$
}

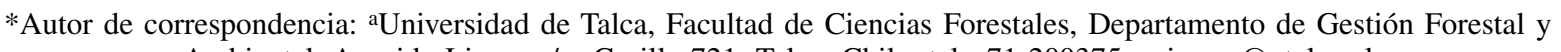
Ambiental, Avenida Lircay s/n, Casilla 721, Talca, Chile, tel.: 71-200375, rpizarro@utalca.cl

\begin{abstract}
SUMMARY
In this research a hydrologic design is defined for infiltration ditches in forest works, for arid and semiarid regions in central Chile. In this context, four plots were evaluated (Hidango, in O'Higgins Region; Name, in Maule Region; and Llohué and Manzanares, in Bío-Bío Region), including two types of infiltration ditches, established in May 2002, linked to a plantation of Pinus radiata. Each plot has a unit witness, which does not present any type of soil treatment. Two types of ditch infiltration were differentiated in width; one of them showed $20 \mathrm{~cm}$ and the other $30 \mathrm{~cm}$, but both possessed the same height of $30 \mathrm{~cm}$. The first one was named ditch type 1 and the other ditch type 2. Results showed that the application of the new hydrologic design for infiltration ditches is very useful when facing extreme events. Also, ditches showed a greater surviving rate for $P$. radiata, being more evident in ditch type 1 that defines less distance between ditches. However, in two plots there is evidence that witnesses showed more biomass than ditches did, although it is not always significant from a statistical point of view. Finally analyzing another type of variables, such as nutrition plant or genetic conditions, becomes necessary to be considered in future research.
\end{abstract}

Key words: infiltration ditch, soil, hydrologic treatment.

\section{RESUMEN}

Esta investigación propuso un diseño hidrológico para la construcción de zanjas de infiltración, en faenas forestales del secano costero e interior de Chile central. Así, se evaluaron cuatro ensayos (Hidango, Región de O'Higgins; Name, Región del Maule; y Llohué y Manzanares, Región del Bío-Bío) con dos tipos de zanjas de infiltración, establecidos en mayo del 2002, y asociados a una plantación de Pinus radiata, con una unidad testigo, la cual no presentaba ningún tipo de tratamiento de suelos. Los dos tipos de zanjas de infiltración se diferenciaron en el ancho, es decir, 20 y $30 \mathrm{~cm}$, y ambas con altura de $30 \mathrm{~cm}$, denominándose zanjas tipo 1 y zanjas tipo 2, respectivamente, elemento que determinó diferentes espaciamientos. Así, a mayor ancho, menor espaciamiento. Los resultados validaron la aplicación de la propuesta de diseño hidrológico de zanjas de infiltración, ratificado por su comportamiento frente a eventos extremos. Asimismo, las zanjas en general favorecieron la sobrevivencia de las plantaciones de P. radiata, especialmente la tipo 1, que define menores distancias de separación entre zanjas. De igual forma, no siempre pareció manifestarse lo mismo en lo referido a la producción de biomasa en los primeros años, dado que existirían otros factores que sería necesario analizar en futuras investigaciones, tales como nutrición de las plantas y selección genética, lo que se manifiesta en que en dos ensayos la situación testigo fue más favorable en biomasa que la situación con zanjas de infiltración, aunque ello no siempre fue significativo desde el punto de vista estadístico.

Palabras clave: zanjas de infiltración, suelos, tratamiento hidrológico.

\section{INTRODUCCIÓN}

En Chile, debido a la conformación geográfica y el tipo de régimen pluviométrico, la erosión hídrica es una de las formas más importantes de degradación del suelo. Así, el que un gran porcentaje de las lluvias precipite en invierno, cuando el suelo está descubierto, unido a malas prácticas de cultivo, provoca importantes daños erosivos (INIA 2001). Es por esto que en Chile, y en el resto del mundo, se reconoce que el proceso erosivo constituye, en términos ambientales y socioeconómicos, el problema de mayor relevancia del sector silvoagropecuario (Francke 1999).

En este marco, la construcción de obras para la captura de aguas de lluvia (water harvesting en la literatura anglosajona), en particular las zanjas de infiltración, ha permitido proporcionar una técnica adecuada para reducir los actuales índices de desertificación, y también propiciar el proceso de infiltración de las aguas de lluvia bajo condiciones edafoclimáticas desfavorables, de tal forma que 
permitan el cultivo o la forestación de zonas de secano (FAO 1994, Boers y Ben-Asher 1982) y la recarga artificial de las napas freáticas.

Existen diferentes estudios ligados al efecto positivo que presentan las zanjas de infiltración, con el objetivo de aumentar la productividad agroforestal en sitios erosionados o degradados (Critchley y Siegert 1991). Uno de ellos, es un estudio comparativo entre dos prácticas de conservación, las zanjas de infiltración y el subsolado, el cual determina que la técnica más recomendable son las zanjas de infiltración, ya que mejoran el establecimiento y permiten aumentar los volúmenes de pino insigne (Pinus radiata D. Don.) hasta cuatro veces (con respecto a un sector sin zanjas), a la edad de 7 años en zonas semiáridas del secano costero de la Región del Maule, Chile (Saavedra 1998). Asimismo, Pérez (2001) estudia el impacto de las zanjas de infiltración en zonas de la Región del Maule, Chile, sobre la productividad de bosques establecidos en dos modalidades: con y sin zanjas de infiltración. El estudio concluye que el impacto de las zanjas de infiltración se traduce en un aumento promedio de un $61 \%$ de los volúmenes totales de madera, en edades de corta entre 18 y 21 años.

Las zanjas de infiltración, en combinación con plantaciones, no sólo permiten la recuperación de terrenos degradados por procesos de erosión y desertificación, sino que han demostrado su alta eficiencia en la captura de humedad, lo que genera un desarrollo más rápido de las plantaciones. Una de las especies más utilizadas en Chile, en conjunto con las zanjas de infiltración, es el $P$. radiata, pero también pueden mencionarse especies tales como eucalipto (Eucalyptus globulus Labill.), eucalipto rojo (Eucalyptus camaldulensis Dehnh.), pimentero (Schinus areira L.), molle (Schinus latifolius [Gill. ex Lindl] Engler), quillay (Quillaja saponaria Mol.), acacio (Robinia pseudoacacia $\mathrm{L}$.), aromo australiano (Acacia melanoxylon $\mathrm{R}$. Brown), nogal (Juglans regia L.) y castaño (Castanea sativa Mill.), como parte de proyectos piloto de biodiversidad (Pizarro et al. 2004a). Así por ejemplo, Faúndez (2004) plantea una experiencia desarrollada en Chile central con pequeños propietarios forestales, y donde se diseñó una zanja de infiltración de dimensiones reducidas y de fácil construcción, en un terreno de 50 hectáreas. El objetivo de esta experiencia fue mejorar la captura del agua de lluvia en el suelo, con fines de alimentación de las napas subterráneas y de favorecer una plantación de $P$. radiata. Como resultado, las zanjas demuestran ser un método muy barato de control de la erosión y de captación de agua de lluvia, constituyéndose en una opción viable para la forestación de terrenos de alta pendiente.

Entre 1993 y 1998 la Corporación Nacional Forestal de Chile (CONAF) y la Agencia de Cooperación Internacional del Japón (JICA) ejecutan el proyecto de Manejo de Cuencas CONAF/JICA "Control de erosión y forestación de cuencas hidrográficas de la zona semiárida de Chile". Este proyecto tiene como propósito el desarrollo y aplicación de técnicas de viverización, forestación y conservación de suelos y aguas, para la recuperación de terrenos degradados por procesos de erosión y desertificación y, entre éstas, se contempla la construcción de zanjas de infiltración. Los resultados del proyecto indican que la combinación de dichas técnicas de control de erosión y de forestación permiten recuperar en forma sostenible zonas rurales degradadas y pauperizadas, lo que posibilita la obtención de estándares de construcción y costos para el establecimiento de estas técnicas en laderas erosionadas (Pizarro et al. 2004a).

En otras experiencias realizadas en América Latina con zanjas de infiltración, se destaca el tratamiento de laderas en Cajamarca, Perú, en zonas de bosque seco montano bajo y de bosque espinoso subtropical, donde los resultados más importantes destacan que la cobertura vegetal en las zonas tratadas con zanjas de infiltración se incrementó de un 10 a un $40 \%$ en tres años; así, en el Parque Demostrativo Aylambo, en años lluviosos con 750 $\mathrm{mm}$ de precipitación anual, la cobertura vegetal herbácea, que inicialmente abarcaba menos del $10 \%$ del área tratada, se incrementa después al $70 \%$. Por otra parte, las zanjas de infiltración se aplican tanto en zonas de cultivo como en zonas de pastos y especies forestales; así se verifica erosión y desbordamiento de agua en el 19\% (zonas de cultivo) y 13\% (zonas de pastos y especies forestales) de los casos, lo cual no significa que la práctica en sí no sea efectiva. Probablemente estos problemas ocurren en zanjas que se encuentran muy alejadas entre ellas o son insuficientes en tamaño. En el 16\% de los casos el distanciamiento entre zanjas varía de 15 a más de $20 \mathrm{~m}$ y en el $84 \%$ restante es de 10 a $15 \mathrm{~m}$. Los resultados muestran que el rendimiento obtenido en pastos y alfalfa, con el empleo de zanjas de infiltración, es mucho mayor que el de las áreas testigo (Carhuapoma y Portugués 1996). En Bolivia, el proyecto JALDA en la ciudad de Sucre valida durante dos años la construcción de zanjas de infiltración (con dimensiones de $2 \mathrm{~m}$ de largo; $0,4 \mathrm{~m}$ de profundidad; $0,4 \mathrm{~m}$ de ancho en la base y $0,5 \mathrm{~m}$ de ancho en la superficie), dentro de unas parcelas agrícolas. Así, se puede constatar que esta práctica es más importante en el control del escurrimiento y en la reducción de la erosión hídrica que en brindar humedad a los cultivos. Los resultados que se obtienen en las parcelas de investigación respecto al rendimiento de los cultivos no muestran ninguna diferencia en favor de los tratamientos con zanjas; en cambio, fue muy notoria la disminución de los síntomas de erosión hídrica por efecto de las zanjas. Esto se evidencia en la gran acumulación de sedimento dentro de las zanjas durante el primer año, el mismo que disminuye notablemente en el segundo año de investigación (Proyecto JALDA 2002).

En la actualidad existen diversos planteamientos de diseño y construcción de obras para la captura e infiltración de aguas lluvias (Villanueva et al. 1987, Giráldez et al. 1988, Hari-Krishna 1989, Boers 1994, Martínez de Azagra 1995), los cuales van desde los modelos más simplistas, que determinan una cierta cantidad de metros lineales de 
zanjas de infiltración por hectárea (CONAF 2005), hasta los modelos más complejos, que incorporan al diseño de la obra el componente hidrológico en un ambiente de microrrelieve y la vegetación existente (Martínez de Azagra 2000). Así, los modelos más simples en general establecen una categorización del proceso de erosión (leve, moderada, grave), y en función de la erodabilidad del suelo se determina la cantidad de metros lineales de zanjas de infiltración que deben ser distribuidas homogéneamente en la ladera, lo cual no siempre puede optimizar la funcionalidad de la zanja de infiltración en términos de la captura, infiltración y disponibilidad del agua lluvia, así como del control del proceso erosivo. Sin embargo, existen planteamientos que consideran a la intensidad de precipitación. Así, Lemus (2003) elaboró un programa computacional, a partir de las aplicaciones de Microsoft Excel, denominado "MAUCO", el cual se fundamenta en la incorporación de un diagnóstico del área degradada, la toma de datos de terreno, la pluviometría, las características de los suelos, la relación relieve-área de mitigación y la cobertura vegetal. Con estos elementos se realiza el diseño de las zanjas de infiltración con el análisis de la precipitación (Lemus 2003). Por otra parte, Martínez de Azagra (1996) desarrolló un modelo computacional, MODIPE, que permite determinar la disponibilidad hídrica o infiltración en un punto de una ladera, la cual se determina para un aguacero aislado o para una serie de aguaceros. Ello obliga a definir las características hidrológicas y topográficas de la ladera, así como las precipitaciones a estudiar. A partir de estos datos, el programa cuantifica la escorrentía superficial generada por los aguaceros y estima la altura de agua que queda residente en un punto de la ladera. Como resultado final se obtiene la cantidad de agua $\left(\mathrm{en} \mathrm{L} / \mathrm{m}^{2}\right)$ disponible en un punto de la ladera. Después de varios años de existencia de dicho programa, se ha constatado la necesidad de incorporar MODIPE a un programa más amplio de diseño de repoblaciones forestales, que no se quede en el dimensionado de algunas técnicas de captura de agua $-\mathrm{y}$ no sólo utilizando para este fin el criterio de economía del agua-, sino que trate también otras cuestiones importantes como, por ejemplo, la densidad de plantación (Mongil 2004).

En este marco, surge la pregunta acerca de cómo debería abordarse el diseño de ingeniería de estas obras, el cual debería incluir aspectos ligados a la ingeniería hidrológica y a la ingeniería de suelos. Ello, debido a que la erosión y sus procesos de cárcavas, movimientos en masa, erosión de manto, torrentes sin control, etc., están determinados, por una parte, por el comportamiento de las precipitaciones (específicamente por la intensidad) y, por otra, por la proporción de escorrentía superficial que se genera en un suelo como producto del proceso precipitación-escorrentía. En virtud de lo anterior, esta investigación generó una metodología basada en ingeniería hidrológica, alternativa a las existentes en el diseño de zanjas de infiltración en distintos sectores de Chile central.

\section{MÉTODOS}

Definición del área de estudio. El área de estudio comprendió al territorio ubicado en el secano costero e interior de Chile central, entre las regiones del Libertador Bernardo O'Higgins, del Maule y del Bío-Bío (figura 1). Allí se instalaron cuatro ensayos experimentales asociados al nuevo diseño y construcción de zanjas de infiltración (cuadro 1), en los cuales se establecieron plantas de $P$. radiata.

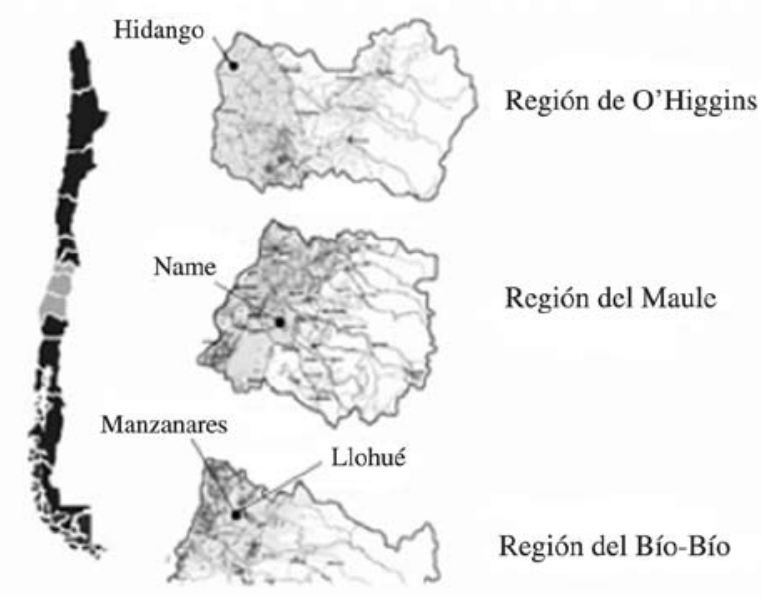

Figura 1. Mapa de ubicación de los ensayos experimentales (Proyecto FDI-CORFO: EIAS. Determinación de estándares de ingeniería en obras de conservación y aprovechamiento de aguas y suelos para la mantención e incremento de la productividad silvícola).

Location map of the experimental sites in central Chile (Project FDI-CORFO: EIAS, Determination of engineering standards in water and soil conservation works for the maintenance and increase of forest productivity).

Cuadro 1. Ubicación espacial de los ensayos con el nuevo diseño hidrológico de zanjas de infiltración.

Location of experimental sites with the new hydrologic design of infiltration ditches.

\begin{tabular}{lcccc}
\hline Ensayo & Región & Norte* & Este* & $\begin{array}{r}\text { Altitud } \\
\text { m s.n.m. }\end{array}$ \\
\hline Hidango & VI & 6.222 .770 & 238.911 & 314 \\
Name & VII & 6.041 .715 & 755.285 & 212 \\
Llohué & VIII & 5.979 .414 & 727.529 & 633 \\
Manzanares & VIII & 5.973 .685 & 723.466 & 97 \\
\hline
\end{tabular}

*Coordenadas UTM (m).

Diseño hidrológico de una zanja de infiltración. El diseño de zanjas de infiltración propuesto atendió fundamentalmente al espaciamiento entre zanjas, de tal forma que permita un control adecuado de la erosión; asimismo, la capacidad de captura de agua de las zanjas debe permitir el almacenaje de un determinado volumen de escorrentía producido por 
la lluvia. En el diseño hidrológico propuesto de zanjas de infiltración se deben considerar previamente cuatro conceptos hidrológicos; el período de retorno, las curvas intensidad-duración-frecuencia, la velocidad de infiltración de los suelos y el coeficiente de escorrentía, para así diseñar las obras que favorecen la infiltración de aguas lluvias en condiciones desfavorables (Pizarro et al. 2004b).

Uno de los primeros requerimientos a considerar fue la determinación del período de retorno $\mathrm{T}$, el que está definido como el tiempo que transcurre entre dos fenómenos de las mismas características, el cual se define por la expresión [1]

$$
T=\frac{1}{1-F(X)}
$$

donde T: período de retorno y $\mathrm{F}(\mathrm{X})$ : es la probabilidad de que la variable aleatoria, intensidad de precipitación, posea un valor menor o igual a un determinado valor $\mathrm{X}$.

Con esto, es posible tener un horizonte de planificación, dentro del cual no debería presentarse un evento con intensidades superiores a la calculada (Pizarro et al. 1986). Asociado a lo que se expone, el período de retorno para el total de ensayos de este estudio correspondió a un $\mathrm{T}=20$ años, el cual se justifica por considerarse este tiempo como el promedio de rotación en plantaciones de P. radiata.

El segundo elemento del diseño fue la determinación y construcción de las curvas Intensidad-Duración-Frecuencia, IDF, curvas que resultan de unir los puntos representativos de la intensidad media en intervalos de diferente duración, y correspondientes todos ellos a una misma frecuencia o período de retorno (Témez 1978). Para ello, se estudió el comportamiento de series de precipitación (superiores a 20 años de datos), a través de una curva que entrega la intensidad media en función de la duración y la frecuencia (figura 2), y cuya única finalidad fue la de aportar patrones de conducta de las lluvias.

La construcción matemática de las curvas IntensidadDuración-Frecuencia (IDF) se realiza, entre otros métodos, según el planteamiento analítico propuesto por Aparicio (1997). Dicho autor plantea la alternativa de obtener una ecuación que genere las curvas IDF a través de un modelo de regresión lineal. Para ello, es necesario relacionar simultáneamente las tres variables en una familia de curvas, para lo cual se utilizó la ecuación [2] propuesta por Aparicio (1997):

$$
I=\frac{k T^{m}}{D^{n}}
$$

donde $\mathrm{k}, \mathrm{m}$ y $\mathrm{n}=$ constantes de regresión lineal múltiple, $\mathrm{T}=$ período de retorno en años, $\mathrm{D}=$ duración en minutos u horas e I = intensidad de precipitación en $\mathrm{mm} / \mathrm{h}$.

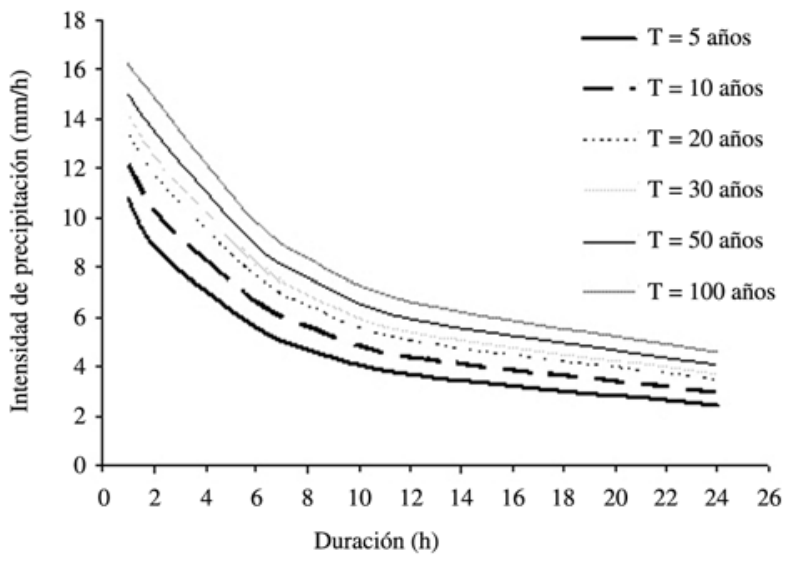

Figura 2. Curva intensidad-duración-frecuencia. Intensity-duration-frequency curve.

Así, es posible obtener un modelo de regresión lineal múltiple para cada zona en que existe una estación pluviográfica. La calidad de la regresión se verificó al analizar ciertas medidas de bondad de ajuste, las cuales corresponden a algunas de regresión no paramétrica, como el coeficiente de determinación $\mathrm{R}^{2}$ y la prueba $\mathrm{U}$ de Mann-Whitney (Gujarati 1992). Si en la zona no se cuenta con estaciones pluviográficas cercanas, es posible aplicar el método K, propuesto por Pizarro et al. (2001), el cual permitió extrapolar las intensidades de precipitación para diversas duraciones horarias, desde estaciones pluviográficas, hacia estaciones pluviométricas cercanas. Para ello, el método considera intensidades máximas de precipitación (en $\mathrm{mm} / \mathrm{h}$ ), para distintos períodos de retorno, entregándose la relación entre la intensidad de precipitación en $1,2,4, \ldots, 12 \mathrm{~h}$, y la intensidad de 24 h, definida como $K=I_{i} / I_{24}$, donde $I_{i}$ corresponde a la intensidad para la duración i,e $\mathrm{I}_{24}$ la intensidad de lluvia para 24 horas. Ello, porque la precipitación de 24 horas es la que se puede encontrar en las estaciones pluviométricas, que cuentan sólo con pluviómetro; así, estas relaciones se traspasan desde la estación pluviográfica a la pluviométrica y permiten construir las curvas IDF en zonas sin datos horarios menores a 24 horas. En este contexto, el presente estudio utilizó las curvas IDF elaboradas por Pizarro et al. (2001).

Un tercer elemento de diseño fue determinar la velocidad de infiltración del agua en el suelo para cada una de las estaciones experimentales. Para ello se utilizó el método del cilindro. Éste permitió obtener registros de las mediciones, en base a una tabla con distintas alturas de agua en el cilindro, a intervalos periódicos, de modo de contar con intervalos a los 5, 10, 20, 30, 45, 60 y 90 minutos (Gurovich 1985). Para la determinación de la velocidad de infiltración [3] se empleó la siguiente expresión de $\mathrm{V}_{\mathrm{i}}$, a saber: 


$$
V_{i}=\frac{D_{h} \cdot 600}{t}
$$

donde, $\mathrm{V}_{\mathrm{i}}=$ velocidad de infiltración en $\mathrm{mm} / \mathrm{h} ; \mathrm{D}_{\mathrm{h}}=$ diferencial de altura de agua en $\mathrm{cm} ; \mathrm{t}=$ diferencial de tiempo en minutos.

Finalmente, el último concepto aplicado al diseño hidrológico fue el coeficiente de escorrentía (e), el cual se define como la parte de la precipitación que se presenta en forma de flujo de agua superficial en el suelo. Su dimensión varía entre 0 y 1 , y está inversamente correlacionado con la capacidad de infiltración, es decir, son superficies impermeables, las que tienen el más alto coeficiente de escorrentía. En esta propuesta metodológica se consideró como recomendable el utilizar valores altos de coeficientes de escorrentía, con el fin de generar mayores condiciones de seguridad, para así garantizar que la obra no será sobrepasada por las aguas lluvias.

En este marco y considerando los valores estándares de coeficiente de escorrentía que se muestran en la literatura hidrológica (Chow et al. 1994, Monsalve 1999), esta investigación propuso que en condiciones de una ladera sobre $15 \%$ de pendiente, descubierta de vegetación y con una erosión moderada (Soto 1997), se utilizara un coeficiente igual a 0,9 , es decir, se considera que casi la totalidad del agua lluvia precipitada en el suelo escurrió $\mathrm{y}$, con ello, se plantea un margen de seguridad importante frente a eventos de envergadura.

En el contexto de los conceptos hidrológicos, el diseño y la construcción de las zanjas de infiltración debe responder a que la cantidad de agua de lluvia que cae desde la zona de impluvio debe ser menor o igual a la que capta y a la que absorbe la zanja, en un diferencial de tiempo, es decir, la capacidad de éstas no debe ser sobrepasada por el total de aportaciones [4] que a ella converjan, a saber:

$$
V_{a z i}=V_{a z z}+V_{a z i n}
$$

donde, $\mathrm{V}_{\mathrm{azi}}=$ volumen de aportación de la zona de impluvio; $\mathrm{V}_{\mathrm{azz}}=$ volumen de captación de la zona de zanjas; $\mathrm{V}_{\mathrm{azin}}=$ volumen de infiltración.

Las zanjas de infiltración construidas (figura 3) poseen un perfil $\mathrm{con} \mathrm{h} \mathrm{cm}$ de altura y $\mathrm{b} \mathrm{cm}$ de base, mientras que éstas tienen un largo 1 variable. El diseño considera además un rebaje en el borde aguas arriba para facilitar la entrada de agua y evitar la erosión de las paredes.

Por otra parte, las expresiones que definen cada una de las variables de la ecuación [4] son desglosadas a partir de la ecuación [5]:

$$
V_{a z i}=I \cdot S \cdot e
$$

donde, $\mathrm{V}_{\mathrm{azi}}=$ volumen de aportación de la zona de impluvio $\left(\mathrm{m}^{3} / \mathrm{h}\right) ; \mathrm{I}=$ precipitación máxima en una hora; $\mathrm{S}=$ superficie de impluvio $\left(\mathrm{m}^{2}\right)$; e $=$ coeficiente de escorrentía.

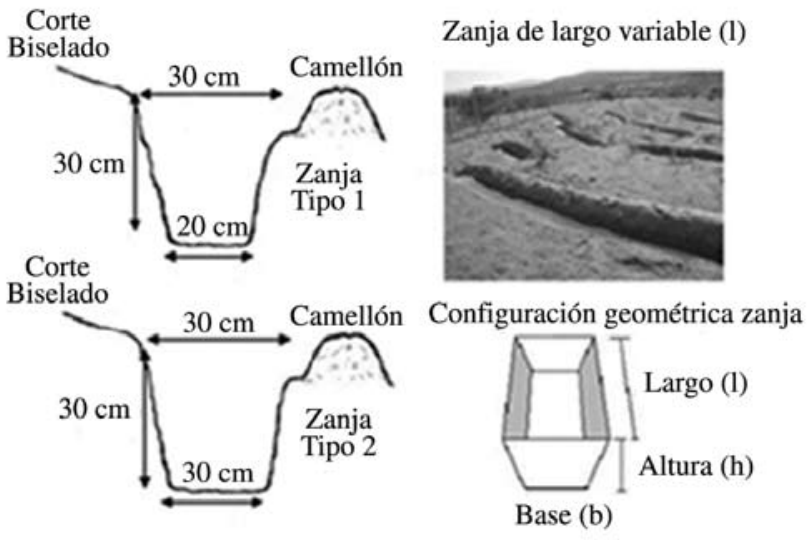

Figura 3. Vista vertical de zanja de infiltración tipo 1 y 2 . Vertical section view of infiltration ditch (type 1 and 2).

Se consideró una hora, porque se asumió que ese período es suficiente para incorporar eventos de alta intensidad al diseño de las obras. El considerar un valor menor a una hora, como es el caso de 30 minutos, involucra costos que a este tipo de obras le es difícil de asumir desde un punto de vista económico, dado que el diferencial de captación e infiltración de agua puede subir en más de un $60 \%$ al considerar 30 minutos y más de un $80 \%$ al incorporar 15 minutos.

Por otra parte,

$$
V_{a z z}=b \cdot h \cdot l
$$

donde $\mathrm{V}_{\mathrm{azz}}=$ volumen de captación de las zanjas $\left(\mathrm{m}^{3} / \mathrm{h}\right)$, la captación en $\mathrm{m}^{3}$ se extiende a una hora para hacer coincidir las unidades; $\mathrm{b}=$ base de la zanja $(\mathrm{m}) ; \mathrm{h}=$ altura de la zanja $(\mathrm{m}) ; 1$ = largo de la zanja $(\mathrm{m})$.

$$
V_{a z i n}=b \cdot v \cdot l
$$

donde $\mathrm{V}_{\mathrm{azin}}=$ volumen de infiltración $\left(\mathrm{m}^{3} / \mathrm{h}\right) ; \mathrm{b}=$ base de la zanja en metros; $\mathrm{v}=$ velocidad de infiltración de la zanja $(\mathrm{m} / \mathrm{h}) ; 1=$ largo de la zanja $(\mathrm{m})$.

Asociado a lo expuesto en la ecuación [4] y operando, se obtiene la siguiente expresión para la distancia horizontal entre zanjas [8]:

$$
\begin{gathered}
V_{a z i}=V_{a z z}+V_{a z i n} \\
I \cdot S \cdot e=(b \cdot h \cdot l)+(b \cdot v \cdot l) \\
I \cdot[l \cdot d] \cdot e=(b \cdot h \cdot l)+(b \cdot v \cdot l) \\
d=\frac{b \cdot(h+v)}{I \cdot e}
\end{gathered}
$$


El distanciamiento horizontal alcanzado permite cumplir la condición inicial de que el volumen de aportes de la zona de impluvio se iguale con la capacidad de captación e infiltración de la zanja. Como este distanciamiento es horizontal, debe ser corregido en función de la pendiente. Asimismo, si el distanciamiento horizontal es conocido y fijo se pueden hacer variar las otras dimensiones, definiendo nuevas alturas y bases, pero manteniendo el principio de igualdad de volúmenes de agua.

A partir de las ecuaciones propuestas, se definieron en los ensayos experimentales distintos espaciamientos horizontales entre zanjas, en función de las características particulares de precipitación y suelo que cada sitio experimental presentó. Esto se asoció a los dos tipos de zanjas utilizados, zanjas tipo 1 (base de $20 \mathrm{~cm}$ y altura de $30 \mathrm{~cm}$ ) y tipo 2 (base de $30 \mathrm{~cm}$ y altura de $30 \mathrm{~cm}$ ), como se muestra en la figura 3. Asimismo, en los ensayos de experimentación se estableció una zona testigo (sin zanjas de infiltración), la cual fue plantada para su posterior evaluación, cuyo esquema general se presenta en la figura 4.

122 metros

\begin{tabular}{|c|cc}
\hline 40 metros & 40 metros & 40 metros \\
\hline \multicolumn{1}{|c|}{ Testigo } & $\begin{array}{c}\text { Zanja 1 } \\
\text { Base }=20 \mathrm{~cm}\end{array}$ \\
& $\begin{array}{c}\text { Zanja 2 } \\
\text { Base }=30 \mathrm{~cm}\end{array}$ \\
\hline
\end{tabular}

Figura 4. Esquema del diseño experimental de los ensayos del proyecto EIAS.

Design of experimental areas in the EIAS project.

Evaluación matemático-estadística del diseño hidrológico de zanjas. La evaluación experimental de los resultados obtenidos se hizo sobre la base de dos variables:

- La respuesta hidrológica de las zanjas: el nuevo diseño plantea que frente a eventos extremos debe cumplirse la condición de que estas obras no deben ser sobrepasadas por el agua de escorrentía que generan los eventos extremos.

- El crecimiento y desarrollo de las plantas: el nuevo diseño debiese asegurar un mejor desarrollo de las plantas de $P$. radiata, en sobrevivencia, altura y diámetro de cuello, donde las últimas dos variables quedan enfundadas en la biomasa promedio producida en cada tratamiento y que se expresa mediante el cálculo del índice de biomasa, el cual se obtuvo a partir del indicador de biomasa [9] (Higueras 2004):

$$
\text { Biomasa }\left(\mathrm{cm}^{3}\right)=\mathrm{D}^{2} \cdot \mathrm{H}
$$

donde $\mathrm{D}=$ diámetro a la altura del cuello $(\mathrm{cm})$; $\mathrm{H}=$ altura total de la planta $(\mathrm{cm})$.
Los resultados obtenidos de las mediciones realizadas a distintos tratamientos con plantas de $P$. radiata se contrastaron con la prueba estadística no paramétrica de Kruskal-Wallis, para discriminar la presencia de diferencias significativas en dichos tratamientos.

Así, se debió establecer la significancia estadística a partir de la siguiente prueba de hipótesis a demostrar:

- Hipótesis nula (Ho): no existen diferencias significativas en el crecimiento en altura y diámetro de cuello de plantas de $P$. radiata en sectores plantados con zanjas de infiltración y un sector testigo, sin obras $(P>0,05)$.

- Hipótesis alternativa (Ha): sí existen diferencias significativas en el crecimiento en altura y diámetro de cuello de plantas de $P$. radiata en sectores plantados con zanjas de infiltración y un sector testigo, sin obras $(P<0,05)$.

De forma adicional se utilizó la prueba no paramétrica de comparación de medianas U de Mann-Whitney, para cada una de las combinaciones posibles de los tratamientos presentes en cada ensayo.

\section{RESULTADOS}

Evaluación hidrológica. Los resultados denotan distanciamientos óptimos, obtenidos bajo los dos tipos de zanjas propuestos en esta investigación (cuadro 2) y, junto con ello, la intensidad máxima horaria registrada y la velocidad de infiltración para cada uno de los cuatro ensayos.

En términos hidrológicos, el nuevo método de diseño propuesto para las zanjas de infiltración permitió que las obras interceptasen y no fuesen sobrepasadas por el agua, lo cual se verificó en terreno de forma clara, ya que frente a fenómenos de alta intensidad de precipitación las obras no fueron sobrepasadas y se cumplieron los supuestos de diseño. Además, se destaca que el año 2002 fue una excelente prueba de validación, dado que se registraron numerosos eventos con altas intensidades de precipitación en la zona de estudio, aunque los equipos instalados para tal fin sólo pudieron entregar información a partir de agosto de 2002. Asimismo, se obtuvieron importantes intensidades de lluvia en los años 2003 y 2004 (cuadro 3), donde paralelamente se observó que las intensidades de diseño en general no fueron sobrepasadas por los valores registrados en terreno, lo que valida el uso de las curvas intensidad-duración-frecuencia.

Por otra parte, la validación hidrológica de las obras se realizó con la información entregada por pluviógrafos instalados en las zonas de los ensayos y que están acompañados de un extractor de datos. Dicha validación se efectuó entre agosto del año 2002 y diciembre del año 2004 (cuadro 3), período en el cual se registraron valores de intensidades horarias de precipitación en general menores a las utilizadas en el diseño y construcción de las obras, 
Cuadro 2. Distanciamiento óptimo entre líneas de zanjas y número de líneas de zanjas dentro de cada ensayo.

Optimal spacing between the rows of ditches and the number of lines of ditches for each test.

\begin{tabular}{|c|c|c|c|c|c|}
\hline \multirow{2}{*}{ Ensayo } & \multicolumn{2}{|c|}{$\begin{array}{l}\text { Distanciamiento entre líneas de zanjas } \\
\text { (metros) }\end{array}$} & \multirow{2}{*}{$\begin{array}{c}\text { Intensidad } \\
\text { máxima } \\
\text { horaria de diseño } \\
(\mathrm{mm} / \mathrm{h})\end{array}$} & \multirow{2}{*}{$\begin{array}{l}\text { Intensidad máxima } \\
\text { registrada en terreno } \\
\qquad(\mathrm{mm} / \mathrm{h})\end{array}$} & \multirow{2}{*}{$\begin{array}{c}\text { Velocidad } \\
\text { de infiltración } \\
(\mathrm{mm} / \mathrm{h})\end{array}$} \\
\hline & $\begin{array}{l}\text { Zanja tipo } 1 \\
\text { (base } 0,2 \mathrm{~m} \text { ) }\end{array}$ & $\begin{array}{l}\text { Zanjas tipo } 2 \\
\text { (base } 0,3 \mathrm{~m} \text { ) }\end{array}$ & & & \\
\hline Hidango & 4,0 & 6,0 & 15,8 & 16,2 & 89,3 \\
\hline Name & 4,0 & 6,0 & 17,6 & 16,2 & 20,3 \\
\hline Manzanares & 4,5 & 6,5 & 19,0 & 9,4 & 72,0 \\
\hline Llohué & 9,0 & 13,0 & 19,0 & 9,4 & 451,3 \\
\hline
\end{tabular}

Cuadro 3. Intensidades máximas de precipitación ( $\mathrm{mm} / \mathrm{h}$ ), en cada zona de estudio (2002-2004).

Maximum rainfall intensity $(\mathrm{mm} / \mathrm{h})$, in each zone of study (2002-2004).

\begin{tabular}{|c|c|c|c|c|c|c|c|c|c|c|c|c|}
\hline Ensayo & Ene & Feb & Mar & Abr & May & Jun & Jul & Ago & Sep & Oct & Nov & Dic \\
\hline \multicolumn{13}{|c|}{ Año 2002} \\
\hline Hidango & - & - & - & - & - & - & - & 9,0 & 5,2 & 9,4 & 1,6 & 1,4 \\
\hline Name & - & - & - & - & - & - & - & 7,4 & 7,6 & 13,6 & 3,0 & 3,6 \\
\hline Manzanares & - & - & - & - & - & - & - & 5,4 & 6,0 & 5,4 & 4,0 & 1,2 \\
\hline Llohué & - & - & - & - & - & - & - & 5,4 & 6,0 & 5,4 & 4,0 & 1,2 \\
\hline \multicolumn{13}{|c|}{ Año 2003} \\
\hline Hidango & 0,4 & 0,2 & 0,2 & 0,2 & 16,2 & 15,0 & 14,4 & 0,0 & 7,2 & 3,6 & 10,2 & 0,8 \\
\hline Name & 3,4 & 0,6 & 0,2 & 1,2 & 8,8 & 10,0 & 10,6 & 3,6 & 4,2 & 4,4 & 10,0 & 0,0 \\
\hline Manzanares & 3,4 & 0,0 & 0,2 & 1,2 & 8,8 & 7,0 & 6,4 & 0,0 & 5,0 & 3,8 & 5,8 & 2,6 \\
\hline Llohué & 3,4 & 0,0 & 0,2 & 1,2 & 8,8 & 7,0 & 6,4 & 0,0 & 5,0 & 3,8 & 5,8 & 2,6 \\
\hline \multicolumn{13}{|c|}{ Año 2004} \\
\hline Hidango & 0,0 & 0,0 & 0,6 & 5,5 & 7,0 & 11,8 & 17,0 & 8,6 & 11,2 & 6,2 & 2,2 & 0,8 \\
\hline Name & 0,6 & 2,8 & 9,4 & 4,0 & 5,0 & 12,2 & 7,0 & 7,0 & 5,4 & 3,4 & 4,4 & 1,2 \\
\hline Manzanares & 0,0 & 1,6 & 9,4 & 6,4 & 3,6 & 7,8 & 5,9 & 3,8 & 6,8 & 5,2 & 5,8 & 2,2 \\
\hline Llohué & 0,0 & 1,6 & 9,4 & 6,4 & 3,6 & 7,8 & 5,9 & 3,8 & 6,8 & 5,2 & 5,8 & 2,2 \\
\hline
\end{tabular}

- : Programación de equipo pluviográfico.

respondiendo favorablemente las zanjas en el proceso de captura e infiltración de agua. Sin embargo, se detectó un valor de intensidad de precipitación de $16,2 \mathrm{~mm} / \mathrm{h}$, registrado en el ensayo de Hidango en el mes de mayo de 2003, que es muy cercano a la información proporcionada por las curvas IDF de la zona de Litueche en la VI Región $(15,8 \mathrm{~mm} / \mathrm{h})$, utilizada como la intensidad de diseño para la construcción de zanjas de infiltración de ese ensayo, lo que no causó daños mayores a la configuración de las zanjas dispuestas en la ladera. Así, se verificó que las zanjas no fueron sobrepasadas en ningún evento y por tanto se validó la metodología desde un punto de vista empírico.

Evaluación del desarrollo y crecimiento de las plantas de P. radiata. En términos de desarrollo y crecimiento de las plantas de $P$. radiata establecidas en los ensayos de experimentación, los resultados del muestreo, al segundo año de establecimiento, evidenciaron aspectos que en general son favorables al uso de las zanjas de infiltración como obras de conservación de aguas y suelos (cuadro 4).

Por otra parte, en la mayoría de los ensayos se pudo observar la diferencia existente entre los testigos y sus respectivos tratamientos (cuadro 4), en que claramente al menos uno de los tratamientos presentó mayor desarrollo en biomasa que los testigos, lo que da cuenta de la efectividad de las obras. En cuanto a la sobrevivencia, los resultados indicaron que la zona con zanjas de infiltración presentó mayores porcentajes de sobrevivencia que la situación testigo, en cada uno de los ensayos, asegurando que la plantación de $P$. radiata asociada a una zanja de infiltración es más eficiente que el establecimiento tradicional.

Análisis estadístico. Tras la aplicación de la prueba estadística no paramétrica de Kruskal-Wallis, fue posible 
Cuadro 4. Evaluación del crecimiento de las plantaciones de $P$. radiata, al segundo año de establecimiento.

Evaluation of the Pinus radiata growth at the second year of establishment.

\begin{tabular}{llccc}
\hline \multirow{2}{*}{ Ensayo } & Tratamiento & $\begin{array}{c}\text { Sobrevivencia } \\
(\%)\end{array}$ & $\begin{array}{c}\text { Biomasa } \\
\text { promedio } \\
\text { por árbol } \pm \\
\text { desviación } \\
\text { estándar } \\
\left(\mathrm{dm}^{3}\right)\end{array}$ & $\begin{array}{c}\text { Productividad } \\
\text { en biomasa } \\
\text { por hectárea } \\
\left(\mathrm{m}^{3} / \mathrm{ha}\right)\end{array}$ \\
\hline Hidango & Testigo & 63,1 & $2,6 \pm 1,8$ & 1,7 \\
& Zanja 1 & 73,1 & $3,9 \pm 2,8$ & 2,3 \\
& Zanja 2 & 66,9 & $5,6 \pm 3,2$ & 3,4 \\
\hline Name & Testigo & 75,0 & $0,7 \pm 0,73$ & 0,4 \\
& Zanja 1 & 88,1 & $1,4 \pm 0,85$ & 0,8 \\
& Zanja 2 & 70,6 & $0,9 \pm 81$ & 0,6 \\
\hline Llohué & Testigo & 83,8 & $5,9 \pm 4,1$ & 4,2 \\
& Zanja 1 & 91,3 & $6,6 \pm 3,7$ & 4,0 \\
& Zanja 2 & 88,8 & $5,0 \pm 3,2$ & 3,1 \\
\hline Manzanares & Testigo & 95,0 & $1,7 \pm 1,3$ & 1,1 \\
& Zanja 1 & 83,8 & $1,6 \pm 1,4$ & 0,9 \\
& Zanja 2 & 100,0 & $3,9 \pm 2,6$ & 1,7 \\
\hline
\end{tabular}

detectar que en todos los casos analizados hubo evidencias de diferencias significativas entre los tratamientos estudiados (zanjas y la unidad testigo): Hidango, $P=0,0000$ (n = 325); Name, $P=0,0000$ ( $=374)$; Llohué, $P=0,0017$ $(\mathrm{n}=422)$ y Manzanares, $P=0,0000(\mathrm{n}=464)$. En este marco, el rechazo de la hipótesis acerca de la igualdad de las distribuciones poblacionales de las cuales habían sido extraídas las muestras generó la necesidad de realizar contrastes que determinaran o precisaran entre qué muestras existían las diferencias significativas que rechazaban la hipótesis nula del contraste de Kruskal-Wallis (Cid et al. 1990, Montgomery 2004). Así, la prueba U de MannWhitney dio como resultado el tratamiento que tiene un mejor efecto en las variables de crecimiento (biomasa).

De acuerdo a los resultados alcanzados, los sectores con plantas de $P$. radiata, habilitados con zanjas, denotaron en la totalidad de los ensayos una mayor sobrevivencia, predominando la zanja tipo 1 , en 3 de los 4 ensayos analizados (cuadro 4). Asimismo, no siempre las zanjas tipo 1 presentaron los mejores resultados que las tipo 2, en lo que respecta a las variables biomasa por hectárea y por árbol. Por último, se apreció que en la zona de Llohué no existía coincidencia en los resultados en biomasa por árbol y por hectárea, como ocurrió en las situaciones restantes y en lo referido a los resultados que alcanzó cada tratamiento.

Desde un punto de vista específico para cada ensayo y considerando los valores de biomasa por planta (cuadro 4),
Cuadro 5. Análisis de diferencias estadísticamente significativas de biomasa entre tratamientos (prueba U de Mann-Whitney).

Significant differences analysis for biomass plants between treatments (U Mann-Whitney test).

\begin{tabular}{llrl}
\hline Ensayos & \multicolumn{1}{c}{ Tratamientos } & Valor $P$ & Decisión \\
\hline Hidango & Testigo-Zanja 1 & 0,0002640 & Rechaza Ho \\
& Testigo-Zanja 2 & $1,850 \mathrm{E}-12$ & Rechaza Ho \\
& Zanjas 1- Zanjas 2 & 0,0000250 & Rechaza Ho \\
\hline Name & Testigo-Zanja 1 & 0,0000000 & Rechaza Ho \\
& Testigo-Zanja 2 & 0,0000850 & Rechaza Ho \\
& Zanjas 1- Zanjas 2 & 0,0000040 & Rechaza Ho \\
\hline Llohué & Testigo-Zanja 1 & 0,0594010 & Rechaza Ho \\
& Testigo-Zanja 2 & 0,1625730 & Acepta Ho \\
& Zanjas 1- Zanjas 2 & 0,0002490 & Rechaza Ho \\
\hline Manzanares & Testigo-Zanja 1 & 0,1462710 & Acepta Ho \\
& Testigo-Zanja 2 & 0,0000000 & Rechaza Ho \\
& Zanjas 1- Zanjas 2 & 0,0000000 & Rechaza Ho \\
\hline
\end{tabular}

se puede plantear lo que sigue: en el ensayo de Hidango, Región de O'Higgins y en el ensayo de Name, Región del Maule, se aprecia que ambos tipos de zanjas superan al testigo, con diferencias significativas (prueba $\mathrm{U}$ de MannWhitney) (cuadro 5).

En el caso de Llohué y Manzanares, Región del Bío-Bío, se da la situación que el testigo es superado por un tipo de zanja. Así, en Llohué la zanja tipo 1 supera al testigo sin diferencias significativas, en tanto que el testigo y las zanjas tipo 1 superan a las zanjas tipo 2 con diferencias significativas. De igual forma, en el caso de Manzanares las zanjas tipo 2 superan con diferencias significativas al testigo y a las zanjas tipo 1; sin embargo, el testigo y las zanjas tipo 1 no presentan entre ellos diferencias significativas.

\section{DISCUSIÓN}

En relación al nuevo diseño hidrológico propuesto para las zanjas de infiltración, la experiencia empírica avala el uso de este método, derivado principalmente de que las zanjas no son sobrepasadas por la escorrentía superficial generada en eventos de alta intensidad en toda la zona de estudio. Ello es esperable, dado que el método se basa en esquemas lógicos de ingeniería hidrológica y define los distanciamientos entre zanjas en base a variables físicas del lugar, más que a recomendaciones sobre territorios muy amplios, lo que la diferencia de otras experiencias (Giráldez et al. 1988, Boers 1994 y Martínez de Azagra 1995). Ello es aún más destacable si se consideran los resultados obtenidos en otras experiencias de terreno en Chile, en donde los fenómenos de desbordamiento de las 
obras son muy frecuentes (Pizarro et al. 2004a). Similares resultados frente a la aplicación de zanjas de infiltración fueron observadas en experiencias de Cajamarca en Perú (Carhuapoma y Portugués 1996), y en Bolivia y en la zona de Sucre (Proyecto JALDA 2002). No obstante, sería interesante utilizar diseños con intensidades de lluvia para períodos menores a una hora, lo cual es posible de alcanzar con las curvas IDF, aunque indudablemente los costos de construcción se verán incrementados en estas obras debido a la necesidad de evacuar mayores volúmenes de agua de escorrentía, lo que incidirá en las dimensiones de las obras (Pizarro et al. 2004b).

Con respecto a los resultados que originaron las plantaciones de $P$. radiata y los efectos observados en los dos tratamientos, surgen diversos aspectos que deben ser discutidos. Así por ejemplo, si se analiza el porcentaje de sobrevivencia de las plantas, en todos los ensayos, éstas muestran un mayor porcentaje si se trata de plantas ligadas a zanjas de infiltración y en donde mayoritariamente (a excepción de una situación) es la zanja tipo 1 la que presenta mejores valores que la tipo 2. La única zona donde las zanjas tipo 2 superan a las tipo 1, es en Manzanares.

En relación a la producción de biomasa se aprecia que en dos de los cuatro ensayos los dos tipos de zanjas superan al testigo, y en los otros dos ensayos, al menos un tipo de zanja supera al testigo (cuadro 5). Asimismo, sólo en uno de los dos ensayos el testigo presenta diferencias significativas con un tipo de zanjas, lo que da cuenta de que existen condiciones locales que demandan un mayor esfuerzo de investigación en estas materias con el fin de obtener mayores niveles de certeza en los resultados. Ello, porque no siempre las zanjas de infiltración parecen superar la situación testigo con respecto a la producción de biomasa, hecho que es similar a lo ocurrido en Bolivia (Proyecto JALDA 2002), aunque sí denotan ser más efectivas en el control de la erosión y en la sobrevivencia de las plantas.

Por último, las distancias definidas entre zanjas, que son mayores en las zanjas tipo 2 que en las tipo 1, no parecen afectar diferencialmente el desarrollo de las plantas, aunque la sobrevivencia parece verse favorecida por un menor distanciamiento entre zanjas y la generación de un menor movimiento erosivo, al reducir la longitud de la ladera.

\section{CONCLUSIONES}

Los resultados alcanzados permiten validar la aplicación de la nueva propuesta de diseño hidrológico de zanjas de infiltración, especialmente por su comportamiento frente a eventos extremos, lo que la diferencia de experiencias previas como las señaladas por otros autores. Asimismo, las zanjas en general favorecen la sobrevivencia de las plantaciones de $P$. radiata, aunque no siempre parece manifestarse lo mismo en lo referido a la producción de biomasa en los primeros años, dado que existirían otros factores condicionantes del desarrollo vegetal, los que sería necesario analizar en futuras investigaciones.

\section{AGRADECIMIENTOS}

Investigación financiada por el Proyecto FDI-CORFO $\mathrm{N}^{\circ}$ 00C7FT-08. A Alejandro Troncoso Aguilar. Instituto de Biología Vegetal y Biotecnología (IBVB) de la Universidad de Talca.

\section{REFERENCIAS}

Aparicio F. 1997. Fundamentos de hidrología de superficie. Balderas, México. Limusa. 303 p.

Boers M, J Ben-Asher. 1982. A review of rainwater harvesting. Agric. Water Manage. 5: 145-158.

Boers M. 1994. Rainwater harvesting in arid and semi-arid zones. Wageningen, Holanda. International Institute for Land Reclamation and Improvement (ILRI). 146 p.

Carhuapoma L, H Portugués. 1996. La agroforestería en Perú. Instituto Nacional de Recursos Naturales, Perú. Consultado 31 mar. 2008. Disponible en http://www.fao.org/regional/ lamerica/redes/sisag/informes/per/.

Chow V, D Maidment, L Mays. 1994. Manual de hidrología aplicada. Santafé de Bogotá, Colombia. Mc Graw-Hill. $584 \mathrm{p}$.

Cid L, C Mora, M Valenzuela. 1990. Inferencia estadística. Concepción, Chile. Universidad de Concepción. 319 p.

Critchley W, K Siegert. 1991. Water harvesting. Manual for the desing and construction of water harvesting schemes for plant production. Roma, Italia. Consultado $31 \mathrm{mar}$ 2008. Disponible en http://www.fao.org/docrep/U3160E/ U3160E00.htm

FAO. 1994. Water harvesting for improved agricultural production. In Food Agriculture Organization of United Nations, Water Reports No 3 (FAO). Roma, Italia. p. 57-71.

Faúndez M. 2004. Análisis comparativo del desarrollo de plántulas de Pinus radiata D. Don. y de la retención del suelo erosionado, en obras de conservación de aguas y suelos. Tesis Licenciatura en Ingeniería Forestal. Talca, Chile. Facultad de Ciencias Forestales, Universidad de Talca. 62 p.

Francke S. 1999. Manejo del suelo posible para el desarrollo forestal del semiárido chileno. Revista Chile Forestal. 7 p. (Documento Técnico $\mathrm{N}^{\circ} 127$ )

Giráldez J, J Ayuso, A García, J López, J Roldán. 1988. Water harvesting strategies in the semiarid climate of southeastern Spain. Agric. Water Manage. 14: 253-263.

Gujarati D. 1992. Econometría. DF México, México. $2^{\mathrm{a}}$ ed. Mc Graw- Hill Latinoamericana. 597 p.

Gurovich L. 1985. Fundamentos y diseño de sistema de riego. San José, Costa Rica. Instituto Interamericano de Cooperación para la Agricultura (IICA). 433 p.

Hari-Krishna J. 1989. Modelling the effects of tied-ridging on water conservation and crop yield. Agric. Water Manage. 16: 87-95.

Higueras P. 2004. Evaluación del crecimiento en una plantación de Eucalyptus globulus Labill con aplicación de fertilizantes 
solubles por medio del riego por goteo, en dos sitios del valle central de la VIII y IX Regiones. Tesis Licenciatura en Ingeniería Forestal. Temuco, Chile. Facultad de Ciencias Agropecuarias y Forestales. Universidad Católica de Temuco. 58 p.

INIA (Instituto Nacional de Investigaciones Agropecuarias, CL). 2001. Diagnóstico sobre el estado de degradación del recurso suelo en el país. Chillán, Chile. Boletín INIA 15. 190 p.

Lemus M. 2003. MAUCO, Programa para el diseño de obras de conservación de suelos. In Seminario Internacional: Restauración Hidrológico-Forestal para conservación y aprovechamiento de aguas y suelos. Santiago, Chile. Consultado 31 mar 2008. Disponible en http://eias.utalca.cl/Seminario/ Exposiciones/13MLemus.pdf.

Martínez de Azagra A. 1995. Modelo para la estimación de las disponibilidades en ladera. Tesis Doctoral. E.T.S.I. Montes. Madrid, España. Universidad Politécnica de Madrid. 324 p.

Martínez de Azagra A. 1996. Diseño de sistemas de recolección de agua para la repoblación forestal. Madrid, España. Mundi-Prensa. 87 p.

Martínez de Azagra A. 2000. Principles for designing endorheic microcatchments. In Third International Congress of European Society for Soil Conservation (ESSC). Valencia, España. Consultado 31 mar 2008. Disponible en http:// www.oasification.com/archivos/Comunicaci\%C3\%B3n\% 20Congreso\%20ESSC.pdf.

Mongil J. 2004. Desarrollo y aplicación de una metodología destinada al dimensionado de sistemas de recolección de agua para la restauración forestal en zonas áridas. Tesis doctor Ingeniero de Montes. Palencia, España. Escuela Técnica Superior de Ingenierías Agrarias de Palencia, Universidad de Valladolid. 381 p.

Monsalve G. 1999. Hidrología en la ingeniería. Santafé de Bogotá, Colombia. $2^{a}$ ed. Escuela Colombiana de Ingeniería. 377 p.

Montgomery D. 2004. Diseño y análisis de experimentos. DF México, México. Grupo Editorial Iberoamérica. 589 p.

Pérez H. 2001. Evaluación de productividad de Pinus radiata (D. Don) asociado a zanjas de infiltración. Llongocura, VII Región del Maule. Tesis Licenciatura en Ingeniería Forestal. Talca, Chile. Facultad de Ciencias Forestales, Universidad de Talca. 72 p.
Pizarro R, P Novoa. 1986. Determinación de valores probabilísticos para variables hidrológicas. In Elementos técnicos de hidrología. Coquimbo, Chile. Corporación Nacional Forestal. 78 p.

Pizarro R, A Abarza, J Flores. 2001. Análisis comparativo de las curvas Intensidad-Duración-Frecuencia (IDF), en 6 estaciones pluviográficas (VII Región del Maule, Chile). Talca, Chile. Consultado 31 mar 2008. Disponible en http://www.unesco. org.uy/phi/libros/analisisMaule.pdf

Pizarro R, C Sangüesa, J Flores, A Martínez, M Ponce. 2004a. Revisión y análisis de prácticas tradicionales de conservación de aguas y suelos en zonas áridas y semiáridas de Chile Central. Talca, Chile. Sociedad de Estándares de Ingeniería para Aguas y Suelos (EIAS). $111 \mathrm{p}$.

Pizarro R, J Flores, C Sangüesa, A Martínez, J García. 2004b. Diseño de obras para la conservación de aguas y suelos. Talca, Chile. Sociedad de Estándares de Ingeniería para Aguas y Suelos (EIAS). 146 p.

Proyecto JALDA. 2002. Zanjas de Infiltración. In Guía técnica sobre el estudio de validación de desarrollo, rural participativo basado en la conservación de suelos y aguas. Sucre. Bolivia. Consultado 31 mar 2008. Disponible en http://www.green. go.jp/gyoumu/kaigai_s/manual/bolivia/index.html.

Saavedra J. 1998. Análisis comparativo de técnicas de recuperación de suelos en áreas degradadas: efecto en la humedad del suelo, la supervivencia y crecimiento de Pinus radiata D. Don. Microcuenca del Estero Barroso, VII Región. Tesis Licenciatura en Ingeniería Forestal. Talca, Chile. Facultad de Ciencias Forestales, Universidad de Talca. 35 p.

Soto G. 1997. Mapa preliminar de la desertificación en Chile - por comunas. Santiago, Chile. Corporación Nacional Forestal CONAF, Ministerio de Agricultura. 88 p.

Témez J. 1978. Cálculo hidrometeorológico de caudales máximos en pequeñas cuencas naturales. Madrid, España. Dirección General de Carreteras. $111 \mathrm{p}$.

Villanueva J, I Sánchez, H Velasco-Molina. 1987. A method to determinate the relation of water harvesting area to planting area on seasonal crops. In Symposium on strategies for classification and management of native vegetation for food production in arid zones. Tucson, Arizona. Estados Unidos. 